\title{
Some Inequalities for Elementary Mean Values
}

\author{
By Burnett Meyer
}

\begin{abstract}
Upper and lower bounds for the difference between the arithmetic and harmonic means of $n$ positive numbers are obtained in terms of $n$ and the largest and smallest of the numbers. Also, results of S. H. Tung [2], are used to obtain upper and lower bounds for the elementary mean values $M_{p}$ of Hardy, Littlewood, and Pólya.
\end{abstract}

1. In 1975, S. H. Tung proved the following theorem [2]:

Let $0<b=x_{1} \leqslant x_{2} \leqslant \cdots \leqslant x_{n}=B$. Let $A$ and $G$ be the arithmetic and geometric means, respectively, of $x_{1}, \ldots, x_{n}$. Then

$$
n^{-1}\left(B^{1 / 2}-b^{1 / 2}\right)^{2} \leqslant A-G \leqslant g(b, B),
$$

where $g(b, B)=c b+(1-c) B-b^{c} B^{1-c}$, and

$$
c=\frac{\log [(b / B-b) \log B / b]}{\log B / b} .
$$

We will derive somewhat similar bounds for the difference between the arithmetic and the harmonic means of $n$ positive numbers.

2. In [1, Chapter 2] Hardy, Littlewood, and Pólya discusséd the elementary mean values, which are defined as follows:

Let $x_{1}, x_{2}, \ldots, x_{n}$ be positive numbers, and let $p$ be a real number. Then $M_{p}\left(x_{1}, \ldots, x_{n}\right)$ is defined as $\left[n^{-1} \sum_{k=1}^{n} x_{k}^{p}\right]^{1 / p}$, if $p \neq 0 ; M_{0}\left(x_{1}, \ldots, x_{n}\right)$ is defined as $\left(\prod_{k=1}^{n} x_{k}\right)^{1 / n}$. We denote $M_{1}$, the arithmetic mean, by $A ; M_{0}$, the geometric mean, by $G$; and $M_{-1}$, the harmonic mean, by $H$. Since $M_{p}\left(k x_{1}, \ldots, k x_{n}\right)=k M_{p}\left(x_{1}, \ldots, x_{n}\right)$ for all $p$ and for all $k>0$, we may, without loss of generality, assume $x_{1}=1$.

THEOREM 1. Let $1=x_{1} \leqslant x_{2} \leqslant \cdots \leqslant x_{n}=B$. Then

$$
\frac{(B-1)^{2}}{n(B+1)} \leqslant A(1, \ldots, B)-H(1, \ldots, B) \leqslant\left(B^{1 / 2}-1\right)^{2} \text {. }
$$

Proof. For each $k, 2 \leqslant k \leqslant n$, let

$$
A_{k}=A\left(x_{1}, x_{2}, \ldots, x_{k-1}, x_{n}\right) \text { and } H_{k}=H\left(x_{1}, x_{2}, \ldots, x_{k-1}, x_{n}\right) \text {. }
$$

Fix $x_{1}, x_{2}, \ldots, x_{n-2}, x_{n}$, and let $x_{n-1}=x$ vary in $[1, B]$. Let

$$
D(x)=A_{n}-H_{n}=\frac{(n-1) A_{n-1}+x}{n}-\frac{n x H_{n-1}}{(n-1) x+H_{n-1}} .
$$

Computation of $D^{\prime}(x)$ shows that $x=H_{n-1}$ is its only positive zero, and standard methods of analysis show that a minimum for $D(x)$ is attained at $x=H_{n-1}$.

Received November 2, 1982; revised February 11, 1983.

1980 Mathematics Subject Classification. Primary 26D20. 
Therefore,

$$
A_{n}-H_{n} \geqslant D\left(\begin{array}{ll}
H_{n} & 1
\end{array}\right)=n^{-1}(n-1)\left(A_{n},-H_{n} \text { 1 }\right) \text {. }
$$

This process may be repeated, giving

$$
\begin{aligned}
A_{n}-H_{n} & \geqslant \frac{n-1}{n}\left(A_{n},-H_{n}\right) \geqslant \frac{n-2}{n}\left(A_{n 2}-H_{n 2}\right) \\
& \geqslant \cdots \geqslant \frac{2}{n}\left(A_{2}-H_{2}\right)=\frac{(B-1)^{2}}{n(B+1)} .
\end{aligned}
$$

The maximum of $D(x)$ must occur at an endpoint. 1 or $B$, as each of the variables $x_{2}, x_{3} \ldots, x_{n}$ in turn varies from 1 to $B$. So

$$
A_{n}-H_{n} \leqslant \frac{n B-(B-1) k}{n}-\frac{n B}{(B-1) k+n}=F(k) .
$$

for some $k, 0 \leqslant k \leqslant n$. The maximum of $F(x)$ on $[0, n]$ will, then. be an upper bound for $A_{n}-H_{n}$. Again, computation of $F^{\prime}(x)$ and standard methods of analysis show that a maximum is attained for $x=n\left(B^{1 / 2}-1\right) /(B-1)$. Hence

$$
A_{n}-H_{n} \leqslant F\left\{n\left(B^{1 / 2}-1\right) /(B-1)\right\}=\left(B^{1 / 2}-1\right)^{2} \text {. }
$$

This completes the proof of Theorem 1.

Upper and lower bounds for $G_{n}-H_{n}$ may be obtained using Theorem 1 and Tung's Theorem. since

$$
G_{n}-H_{n}=\left(A_{n}-H_{n}\right)-\left(A_{n}-G_{n}\right)
$$

3. Tung's Theorem may be used to obtain upper and lower bounds for the elementary mean values $M_{p}$. by using the relation

$$
M_{p}\left(x_{1} \ldots \ldots x_{n}\right)=\left\{A\left(x_{1}^{p} \ldots \ldots x_{n}^{p}\right)\right\}^{1 / p}
$$

(See [1].)

TheOREM 2. Let $1=x_{1} \leqslant x_{2} \leqslant \cdots \leqslant x_{n}=B$. and let $p>0$. Then

$$
\left[n^{-1}\left(B^{p / 2}-1\right)^{2}+G^{p}\right]^{1 / p} \leqslant M_{p}(1 \ldots B B) \leqslant\left[g\left(1, B^{p}\right)+G^{p}\right]^{1 / p} .
$$

where $G=G(1, \ldots B)$, and $g$ is the function defined in Tung's Theorem.

TheOREM 3. Let $1=x_{1} \leqslant x_{2} \leqslant \cdots x_{n}=B$, and let $p<0$. Then

$$
\left[g\left(B^{p}, 1\right)+G^{p}\right]^{1 / p} \leqslant M_{p}(1 \ldots B) \leqslant\left[n^{-1}\left(1-B^{p / 2}\right)^{2}+G^{p}\right]^{1 / p} .
$$

where $G=G(1, \ldots B)$ and $g$ is the function of Tung 's Theorem.

Department of Mathematics

University of Colorado

Boulder. Colorado 8()30)

1. (i. H. Hardy, J. E. Lithliwood \& (i. Poilya, Inequalities, Cambridge Univ. Press, Cambridge. 1952.

2. S. H. TUNG. "On lower and upper bounds of the difference between the arithmetic and the geometric mean." Math. Comp.. v. 29. 1975, pp. 834-836. 